\title{
BREAKDOWN OF HOMOGENIZATION FOR THE RANDOM HAMILTON-JACOBI EQUATIONS*
}

\author{
WEINAN E $E^{\dagger}$, JAN WEHR ${ }^{\ddagger}$, AND JACK XIN§
}

\begin{abstract}
We study the homogenization of Lagrangian functionals of Hamilton-Jacobi equations (HJ) with quadratic nonlinearity and unbounded stationary ergodic random potential in $R^{d}, d \geq 1$. We show that homogenization holds if and only if the potential is bounded from above. When the potential is unbounded from above, homogenization breaks down, due to the almost sure growth of the running maxima of the random potential. If the unbounded randomness appears in the advection term, homogenization may or may not hold depending on the structure of the flow field. In (compressible) unbounded gradient flows, homogenization holds in spite of the unboundedness. In (incompressible) unbounded shear flows, homogenization breaks down again due to unbounded running maxima of the flows. Results for random advection follow from a transformation of the problem to that of HJ with random potential. Analogous effective behavior is present for front speeds in reaction-diffusion-advection equations with unbounded random advection, and may have broader implications for wave propagation in random media.
\end{abstract}

Key words. random Lagrangian functionals, homogenization, divergence, extreme behavior

AMS subject classifications. 76M50, 60H05, 76M35

\section{Introduction}

Stochastic Hamilton-Jacobi equations (HJ) appear as useful prototype models for wave propagation and growth phenomena in random media, see [3, 14, 15] among others. The effective large space-time behavior can be captured by studying the homogenization limit $(\epsilon \rightarrow 0)$ of the associated random Lagrangian functional:

$$
S^{\epsilon}(x, t, \omega)=\inf _{\xi \in A} \int_{0}^{t} L\left(\xi(s) / \epsilon, \xi^{\prime}(s), \omega\right) d s,
$$

where $A=\left\{\xi \in W^{1, \infty}\left([0, t] ; R^{d}\right): \xi(0)=0, \xi(t)=x\right\} ; L=L(x, q, \omega), x, q \in R^{d}(d \geq 1)$, is the Lagrangian (Legendre transform) of a convex random Hamiltonian $H(x, p, \omega)$ to be specified. The earlier works $[11,10]$ showed that if the Lagrangian (Hamiltonian) is convex in $q(p)$ with superlinear growth in large $|q|(|p|)$, and uniformly bounded in $x$, then $S^{\epsilon}$ converges almost surely to a deterministic function, $S^{*}(x, t)=t \mu^{*}\left(\frac{x}{t}\right)$, where $\mu^{*}=\mu^{*}(q)$ is a convex function with superlinear growth in $q$. The Legendre transform of $\mu^{*}$ gives the effective Hamiltonian $H^{*}=H^{*}(p)$ which defines the homogenized HJ equation $u_{t}+H^{*}\left(\nabla_{x} u\right)=0$. For affine initial data $u(x, 0)=p \cdot x$, the solution is a propagating front $u(x, t)=p \cdot x-H^{*}(p) t$, where $H^{*}(p)$ is the effective front speed in unit direction $p$.

In this paper, we consider Hamiltonians or Lagrangians which are unbounded in $x$; for example, the $x$ dependence can be an unbounded Gaussian process. We are interested in the existence of the homogenization limit (1.1). It turns out that the existence of the limit is quite subtle because of the unbounded randomness. When the Hamiltonian is of the form: $H(x, p, \omega)=|p|^{2} / 2+V(x, \omega), p, x \in R^{d}$,

\footnotetext{
${ }^{*}$ Received: September 17, 2007; accepted: November 11, 2007. Communicated by Shi Jin.

${ }^{\dagger}$ Department of Mathematics, Princeton University, Princeton, NJ 08544, USA (weinan@ math.princeton.edu).

${ }^{\ddagger}$ Department of Mathematics, University of Arizona, Tucson, AZ 85721, USA (wehr@math.ari zona.edu).

${ }_{\S}^{\S}$ Department of Mathematics, UC Irvine, Irvine, CA 92697, (jxin@math.uci.edu).
} 
the homogenization limit exists if and only if the random potential is bounded from above. In the case when the Hamiltonian contains a compressible random advection, $H(x, p, \omega)=|p|^{2} / 2+p b(x, \omega), x, p \in R^{1}$, the homogenization limit holds irrespective of the unboundedness of $b(x)$. We shall present elementary proofs of these statements. Similar findings exist for random front speeds in related Burgers and reactiondiffusion-advection equations $[4,12,13,9]$. Hence the phenomena demonstrated here may have broad implications for wave propagation in random media.

A simple scaling transform $\epsilon=\frac{1}{n}$ shows that (1.1) is equivalent to studying the limit $S_{n}(x, t) / n$, where:

$$
S_{n}(x, t)=\inf _{\xi \in A_{n}} \int_{0}^{n t} L\left(\xi(s), \xi^{\prime}(s), \omega\right) d s,
$$

and $A_{n}=\left\{\xi \in W^{1, \infty}\left([0, n t] ; R^{n}\right): \xi(0)=0, \xi(n t)=n x\right\}$.

The plan for the paper is as follows. In Section 2, we will show that the limit (1.2) exists and is deterministic for the quadratic Hamiltonian $H(x, p, \omega)=|p|^{2} / 2+V(x, \omega)$ for a class of random potentials $V$ which are bounded above. By a transformation, we prove homogenization for the Hamiltonian $H(x, p, \omega)=|p|^{2} / 2+b(x, \omega) \cdot p$, where $b$ is a random gradient field with appropriate smoothness properties.

More precisely, the homogenization problem in the case of a gradient flow is reduced to that of a Hamiltonian of the type mentioned above with a random potential $V(x)=-\frac{1}{2}|b|^{2}(x)$, which is always bounded above. In Section 3, we study a general class of random potentials unbounded from above in $R^{d}$, and show that homogenization breaks down. Instead, the behavior of the system on each length scale is dominated by the maximum of the potential on this scale. The Hamiltonian of shear flows is reduced to such a regime as well.

We thus see through these case studies that large space-time effects of randomness can take two very different forms:

1. Homogenization. In this case, the behavior of the system on large scales is described by an effective Lagrangian (or Hamiltonian) which is nonrandom. The disorder gets averaged, and the extreme nature of the random media is tamed. An elementary (and linear) analog of this phenomenon in classical probability theory is the strong law of large numbers [2].

2. Domination by finite-volume maxima of the random potential. In this case, homogenization breaks down, and on an arbitrarily large scale, the behavior of the system is dictated by the maximum value of the disorder on that scale. The extreme nature of the random media prevails. A relevant problem of classical probability theory is the study of extrema of stochastic sequences and processes [6]. For example, the maximum $M_{n}$ of $n$ independent unit normal random variables behaves asymptotically as $\sqrt{2 \log n}$ and, in particular, diverges as $n \rightarrow \infty$. We will see that similar divergence of maxima of random fields underlies the phenomenon described here.

The two types of behavior of random systems are incompatible with each other. For the class of Hamilton-Jacobi equations studied here, the behavior depends on a simple mathematical criterion: boundedness of the potential (or transformed potential) from above. The one-sided boundedness of spatial potential is the sharp extension of the boundedness assumption in $[11,10]$.

A discussion of the equivalence of Hamiltonians and Lagrangians in the sense of homogenization is given in Section 4. In particular, such equivalence means that the minimizing paths of their Lagrangians obey the same equation of motion, and 
that their action functionals differ asymptotically by an amount independent of the particle paths connecting the same initial and end points.

If the Hamiltonian is also random in time, then temporal fluctuations with finite correlation lengths may promote mixing and facilitate averaging (homogenization). Recently, existence and estimates of front speeds in unbounded temporal random flows have been studied for reaction-diffusion-advection equations [13, 7, 9]. It is worthwhile for a future work to study whether HJ averaging in unbounded time-random regime behaves similarly.

\section{Potential bounded from above and homogenization}

2.1. Hamiltonians in classical mechanics. Let $V(x), x \in R^{d}$ be a stationary, ergodic random field, such that, for a certain constant $V_{0}, V(x) \leq V_{0}$ with probability one. Here ergodicity means ergodicity with respect to the translation group $R^{d}$. We assume that $\mathbf{E}[|\mathbf{V}(\mathbf{x})|]<\infty$.

Let us consider the Hamiltonian $H(x, p)=\frac{p^{2}}{2}+V(x)$ of a classical particle moving in the field of the potential $V$. The corresponding Lagrangian is $L(x, q)=\frac{|q|^{2}}{2}-V(x)$. Let us fix $x \in R^{d}$ and $t>0$. The action integral for a particle moving from the origin to the point $n x$ in time $n t$ along the path $s \mapsto \xi(s), 0 \leq s \leq n t$ equals

$$
\Phi_{n}=\int_{0}^{n t} L(\xi(s), \dot{\xi}(s)) d s .
$$

We study the minimum $S_{n}(t, x)$ of $\Phi_{n}$ over all paths $\xi$ such that $\xi(0)=0$ and $\xi(n t)=$ $n x$.

Proposition 2.1. There exists a nonrandom number $S^{*}(t, x)$ such that with probability one

$$
\lim _{n \rightarrow 0} \frac{S_{n}(t, x)}{n}=S^{*}(t, x)
$$

Proof. We apply Kingman's subadditive ergodic theorem $[2,10]$ to the family $S_{m, n}(t, x)$, where $m$ and $n$ are nonnegative integers such that $m<n$, defined as

$$
S_{m, n}(t, x)=\min _{\xi(m t)=m x ; \xi(n t)=n x} \int_{m t}^{n t} L(\xi(t), \dot{\xi}(t)) d t,
$$

with the minimum taken over all paths $\xi$ connecting $m x$ to $n x$ in the time $(n-m) t$. The theorem $[2,10]$ says the following. Suppose that $S_{m, n}$ are random variables satisfying:

(1) $S_{0,0}=0, S_{m, n} \leq S_{m, k}+S_{k, n}$, for $m \leq k \leq n$;

(2) $\left\{S_{m, m+k}, m \geq 0, k \geq 0\right\}$ equals $\left\{S_{m+1, m+k+1}, m \geq 0, k \geq 0\right\}$ in distribution;

(3) $E\left[S_{0,1}^{+}\right]<+\infty ; \alpha_{n} \equiv E\left[S_{0, n}\right]<\infty$;

then the following hold:

(1) $\alpha=\lim _{n \rightarrow \infty} \frac{\alpha_{n}}{n}=\inf _{n \geq 1} \frac{\alpha_{n}}{n} \in[-\infty, \infty)$; (2) $S_{\infty}=\lim _{n \rightarrow \infty} \frac{S_{0, n}}{n}$ exists with probability one;

(3) if $E\left[S_{\infty}\right]=\alpha>-\infty, \lim _{n \rightarrow \infty} E\left[\left|S_{0, n} / n-S_{\infty}\right|\right]=0$. 
For $l<m<n$, we have clearly from $(2.2)$ :

$$
S_{0, m}(t, x)+S_{m, n}(t, x) \geq S_{0, n}(t, x) .
$$

Next, for a linear path $\xi(s)=s \frac{x}{t}$, we obtain

$$
\Phi_{n}=\frac{n|x|^{2} t}{2}-\int_{0}^{n t} V\left(\frac{s x}{t}\right) d s,
$$

which, by the assumption of integrability of $V(x)$, implies that

$$
\mathbf{E}\left[\left|S_{0, n}(t, x)\right|\right]<+\infty \text {. }
$$

On the other hand, since the kinetic part of the action integral is positive, the upper bound on $V$ implies that

$$
\mathbf{E}\left[S_{0, n}(t, x)\right] \geq-n t V_{0}
$$

The family $S_{m, n}$ thus satisfies the assumptions (1)-(3) of the subadditive ergodic theorem, implying existence of the finite limit of $\frac{S_{0, n}}{n}$. This limit is invariant under translations of the realization of $V$ by vectors proportional to $x$; hence its value is constant with probability one.

2.2. Advection in gradient flows. Let us consider Hamiltonians of the form $H(x, p)=\frac{p^{2}}{2}+p \cdot b(x), p, x \in R^{d}$. Here $b(x)=\nabla U(x)$, where $U(x)$ is a scalar random vector field whose realizations are of class $\mathbf{C}^{2}$, and let us assume that the scalar random field

$$
V_{1}(x)=-\frac{1}{2}|b|^{2}(x)
$$

satisfies all the conditions on classical potentials in the previous subsection (clearly, $V_{1}$ is bounded above). Examples of such fields $b$ include Gaussian random fields with appropriate covariance.

The Lagrangian corresponding to the above $H$ is

$$
L(x, q)=\frac{1}{2}|q-b(x)|^{2}=\frac{|q|^{2}}{2}-q \cdot b(x)+\frac{|b(x)|^{2}}{2} .
$$

As in the previous section, consider a path $\xi(s), 0 \leq s \leq n t$, such that $\xi(0)=0$ and $\xi(n t)=n x$. For such a path, the contribution from the second term to the Lagrangian integral equals

$$
\int_{0}^{n t} b(\xi(s)) \cdot \dot{\xi}(s) d s=U(n x) .
$$

This shows that this term is a null Lagrangian, i.e., that the Lagrangian (2.3) leads to the same Euler-Lagrange equations of motion as the Lagrangian of the potential system:

$$
L_{1}(x, q)=\frac{|q|^{2}}{2}-V_{1}(x)
$$

where $V_{1}(x)=-\frac{1}{2}|b|^{2}(x)$. Thus the result of the previous section applies and implies that the homogenization holds for such Hamiltonian of advection type, even though the flow field is unbounded. 


\section{Unbounded potentials and domination by finite-volume maxima}

In this section we consider again a particle moving in a potential $V$, which is a realization of a stationary random field, but this time the support of the distribution of $V(x)$ is unbounded from above. We also assume that the covariance of $V$ decays sufficiently fast. We will show that unboundedness of $V$ leads to a different behavior of the system, where the homogenization limit fails.

3.1. One dimensional potentials. Consider a particle moving in a potential field $V$ in one dimension, according to the equations of classical mechanics. Let $V$ be a realization of a stationary random process. For simplicity, we take $V$ to be the Ornstein-Uhlenbeck process, i.e., the Gaussian process with mean zero and the covariance

$$
\mathbf{E}[V(x) V(y)]=\frac{1}{2} \exp (-|x-y|) .
$$

Let us fix $x, t>0$. For any $n$, the trajectory of the particle, starting from the point 0 at time $t=0$ and arriving at the point $n x$ at time $n t$, has the classical action equal to:

$$
S_{n}=\min \int_{0}^{n t}\left[\frac{1}{2}\left(\frac{d u}{d \tau}\right)^{2}-V(u(\tau))\right] d \tau,
$$

where the infimum is taken over all $C^{2}$ functions $u(\tau)$ such that $u(0)=0$ and $u(n t)=$ $n x$. Our goal is to study the asymptotic behavior of $\frac{S_{n}}{n}$ as $n \rightarrow \infty$. Clearly, the function $u$, which minimizes the action integral, is monotone increasing and satisfies $\frac{d u}{d \tau} \neq 0$ for all $\tau$.

We can thus rewrite the action in terms of the minimum over the inverse functions $\tau(u)$ :

$$
S_{n}=\min \int_{0}^{n x}\left[\frac{1}{2}\left(\frac{d \tau}{d u}\right)^{-1}-V(u) \frac{d \tau}{d u}\right] d u
$$

where the minimum is over all $C^{2}$ functions $\tau(u)$ such that $\tau(0)=0$ and $\tau(n x)=n t$. It follows from the principle of energy conservation that the solution of the above variational problem satisfies

$$
\frac{d \tau}{d u}=\frac{1}{\sqrt{2\left[E_{n}-V(u)\right]}},
$$

where $E_{n}$ is the total energy of the particle and therefore

$$
S_{n}=\int_{0}^{n x}\left[\sqrt{\frac{E_{n}-V(u)}{2}}-\frac{V(u)}{\sqrt{2\left(E_{n}-V(u)\right)}}\right] d u
$$

and

$$
\int_{0}^{n x} \frac{1}{\sqrt{2\left(E_{n}-V(u)\right)}} d u=n t .
$$

In particular, $E_{n}>V(u)$ for all $u \in[0, n x]$ for the particle to be able to reach the point $n x$. We rewrite the formula for $S_{n}$ as follows:

$$
S_{n}=\int_{0}^{n x}\left[\sqrt{\frac{E_{n}-V(u)}{2}}+\frac{E_{n}-V(u)}{\sqrt{2\left(E_{n}-V(u)\right)}}-\frac{E_{n}}{\sqrt{2\left(E_{n}-V(u)\right)}}\right] d u .
$$


The first two terms in the integrand are identical and their integral can be estimated by Jensen's inequality (using concavity of the root function):

$$
\frac{1}{n x} \int_{0}^{n x} \sqrt{2\left(E_{n}-V(u)\right)} d u \leq \sqrt{\frac{1}{n x} \int_{0}^{n x} 2\left(E_{n}-V(u)\right) d u}=\sqrt{2 E_{n}-2 \frac{1}{n x} \int_{0}^{n x} V(u) d u} .
$$

By ergodicity of the Ornstein-Uhlenbeck process, we have

$$
\frac{1}{n x} \int_{0}^{n x} V(u) d u \rightarrow 0
$$

with probability 1 as $n \rightarrow \infty$ and therefore the integral of the first two terms in the expression for $S_{n}$ is bounded by $3 \sqrt{E_{n}} n x$ for large $n$. The integral of the third term is simply $-E_{n} n t$, so for large $n$ we obtain

$$
-t E_{n} \leq \frac{1}{n} S_{n} \leq 3 x \sqrt{E_{n}}-t E_{n}
$$

As mentioned above, $E_{n} \geq \sup _{u \in[0, n x]} V(u)$. Since the realizations of the OrnsteinUhlenbeck process $V(u), u \geq 0$, are unbounded from above with probability one, it follows that $\frac{S_{n}}{-n t E_{n}} \rightarrow 1$ with probability 1 . In particular, $\frac{S_{n}}{n}$ is unbounded, which implies that the Hamilton-Jacobi equation with the potential $V$ does not homogenize in the usual sense $[11,10]$. Moreover, since for $E_{n}>\sup _{u \in[0, n x]} V(u)+\frac{x^{2}}{2 t^{2}}$ we have $\int_{0}^{n x} \frac{1}{\sqrt{2\left(E_{n}-V(u)\right)}} d u<n t$, it follows from the condition satisfied by $E_{n}$ that $E_{n} \leq$ $\sup _{u \in[0, n x]} V(u)+\frac{x^{2}}{2 t^{2}}$. Consequently, $S_{n}$ is asymptotically equivalent to $-t V^{*}(n x)$, where $V^{*}(y)=\sup _{u \in[0, y]} V(u)$ is the running maximum of the Ornstein-Uhlenbeck process. Asymptotic equivalence of the two sequences means that their ratio converges to a positive constant.

We will invoke the following classical result about the running maximum of the Ornstein-Uhlenbeck process. It follows from the one dimensional versision of Theorem 6.9.5 in chapter 6 of [1].

THEOREM 3.1. The running maximum of $V^{*}(y)$ of the Ornstein-Uhlenbeck process satisfies the limit theorem:

$$
\operatorname{Prob}\left[\frac{V^{*}(y)-b_{y}}{a_{y}} \leq x\right] \rightarrow \exp \left(-e^{-x}\right)
$$

as $y \rightarrow \infty$, where

$$
a_{y}^{-1} \sim b_{y} \sim(2 \log y)^{\frac{1}{2}},
$$

with $\sim$ denoting asymptotic equivalence.

The theorem says that the renormalized random variables $V^{*}(y)$ converge in distribution to the double exponential distribution. It follows that

$$
\frac{V^{*}(y)}{(2 \log y)^{\frac{1}{2}}} \rightarrow 1, \text { as } y \rightarrow \infty,
$$

in probability, implying that

$$
\frac{S_{n}}{n} \rightarrow-\infty
$$


in probability.

The standard homogenization limit fails as claimed. Instead, the modified limit holds in probability:

$$
\frac{S_{n}}{n(2 \log n)^{1 / 2}} \rightarrow-t
$$

The divergence of homogenization in particular means that for affine data, the growth rate of HJ solutions in time is faster than linear. We shall come back to this point for front solutions in Section 3.3.

Generalization to other random potentials is straightfoward. In the above analysis, we have only used the fact that $V$ is a mean-zero Gaussian process to which Theorem 6.9 .5 of [1] applies. For example, the divergence results hold for stationary Gaussian processes with the covariance function $r(\tau)$ satisfying

$$
r(\tau)=1-C|\tau|^{\alpha}+o\left(|\tau|^{\alpha}\right)
$$

as $\tau \rightarrow 0$ for some $\alpha \in(0,2]$, and $r(\tau) \in L^{2}\left(R^{1}\right)$. The Ornstein-Uhlenbeck process satisfies this condition with $\alpha=1$. Processes which satisfy it with $\alpha=2$ have differentiable realizations.

3.2. Unbounded potential in several dimensions. We show that the behavior of a particle in a multi-dimensional random potential unbounded from above is similar to that in one dimension. As before, we study the action $S_{n}$ of the particle which goes from the origin to the point $n x$ in time $n t$, where $x$ is a fixed vector in $\mathbf{R}^{\mathbf{d}}$. Let $x_{n}^{*}$ denote the point in the ball $B(0, n|x|)$ where $V$ reaches its maximum, equal to $V_{n}^{*}$. We also denote the minimum of $V$ in the same ball by $V_{* n}$. To obtain an upper bound on $S_{n}$, consider a path which first goes from the origin to $x_{n}^{*}$ in time $\delta n t$, moving with a constant velocity (equal to $\frac{x_{n}^{*}}{\delta n t}$ ), next spends time $(1-2 \delta) n t$ at $x_{n}^{*}$ and finally goes from $x_{n}^{*}$ to $n x$ in the remaining time $\delta n t$, moving with the constant velocity $\frac{n x-x_{n}^{*}}{\delta n t}$. For such paths, we get:

$$
S_{n} \leq \frac{1}{2} \delta n t\left(\frac{\left|x_{n}^{*}\right|}{\delta n t}\right)^{2}-\delta n t V_{* n}-(1-2 \delta) n t V_{n}^{*}+\frac{1}{2} \delta n t\left(\frac{\left|n x-x_{n}^{*}\right|}{\delta n t}\right)^{2}-\delta n t V_{* n}
$$

It follows that

$$
\frac{S_{n}}{n} \leq \frac{5}{2} \frac{|x|^{2}}{\delta t}-2 \delta t V_{* n}-(1-2 \delta) t V_{n}^{*}
$$

Because the process has mean zero, $V_{* n}=-V_{n}^{*}$ in distribution. Applying the asymptotic law of $V_{n}^{*}$ (Theorem 6.9.5, [1]), we have the upper bound:

$$
\frac{S_{n}}{n} \leq \frac{5|x|^{2}}{\delta t}-\frac{1}{4} t V_{n|x|}^{*}
$$

for $\delta$ small enough. By almost sure logarithmic divergence of $V_{n|x|}^{*}$ as $n \rightarrow \infty$ (true for a Gaussian process), we conclude that $S_{n} / n \rightarrow-\infty$ almost surely, and standard homogenization limit again fails. It will be interesting to establish a precise divergence result as in the one space dimensional case. However, the source of divergence is the same: dominance of the running maxima of an unbounded random process. 
3.3. Front divergence in shear flows. Let $b(x)=\left(V_{2}\left(x^{\prime}\right), 0\right), x^{\prime}=$ $\left(x_{2}, \cdots, x_{d}\right), 0 \in R^{d-1}$, namely a shear flow in the direction $x_{1}$. The HJ equation associated with the Hamiltonian $H(x, p)=|p|^{2} / 2+b \cdot p$ is:

$$
u_{t}+b(x) \cdot \nabla_{x} u+\left|\nabla_{x} u\right|^{2} / 2=0 .
$$

Consider a solution that represents a front moving in the $x_{1}$ direction in the form $u(x, t)=x_{1}-\frac{1}{2} t+w\left(x^{\prime}, t\right)$. Then $w$ satisfies the HJ equation:

$$
w_{t}+\left|\nabla_{x^{\prime}} w\right|^{2} / 2+V_{2}\left(x^{\prime}\right)=0
$$

which is in the classical potential form. If $V_{2}$ obeys the assumptions in previous subsections and is unbounded from above, $-w\left(x^{\prime}, t\right) / t$ diverges for large time $t$, and the front speed is not asymptotically constant. Instead there exists front speed accelaration due to the dominance of running maxima of process $V_{2}$. Shear flow is a special incompressible (divergence free) flow. It is interesting to study other types of random incompressible flows that may lead to divergence.

4. Discussion of Hamiltonians and Lagrangians

The equation of motion for the Hamiltonian $H_{1}=\frac{p^{2}}{2}+b(x) p, x, p \in R^{1}$, is:

$$
\xi^{\prime \prime}(s)=b(\xi(s)) b^{\prime}(\xi(s)) .
$$

As discussed earlier, the same equation also arises from another Hamiltonian: $H_{2}=$ $\frac{p^{2}}{2}+V_{1}(x)$, where $V_{1}(x)=-\frac{b^{2}(x)}{2}$. In this sense, the advection model is equivalent to a potential model, and homogenization of the two models is closely related.

In the study of homogenization of the related partial differential equations, we are not only interested in the ordinary differential equations satisfied by the minimizing paths, but also in the asymptotic behavior of the corresponding action integrals. The Lagrangians corresponding to $H_{1}$ and $H_{2}$ (their Legendre transforms) are respectively

$$
L_{1}(x, q)=\frac{1}{2}(q-b(x))^{2}
$$

and

$$
L_{2}(x, q)=\frac{1}{2}\left(q+b^{2}(x)\right) .
$$

That the Hamiltonians $H_{1}$ and $H_{2}$ lead to the same equations of motion implies that the difference of the corresponding action functionals,

$$
\int_{0}^{n t}\left[L_{2}(\xi(s), \dot{\xi}(s))-L_{1}(\xi(s), \dot{\xi}(s))\right] d s,
$$

does not depend on the path $\xi(s)$. Taking the linear function $\xi(s)=\frac{x}{t} s$, we obtain:

$$
\int_{0}^{n t}\left[L_{2}(\xi(s), \dot{\xi}(s))-L_{1}(\xi(s), \dot{\xi}(s))\right] d s=\int_{0}^{n x} b(u) d u .
$$

Divided by $n$, this expression converges with probability one to the expected value of $b(0)$ times $x$. Consequently, homogenization or breakdown of homogenization holds for $H_{1}$ and $H_{2}$ simultaneously. In a general classical mechanics Hamiltonian (as well as in a shear flow Hamiltonian), the potential can be unbounded from above. In contrast, the transformed potentials from the gradient flow Hamiltonians are nonpositive. 


\section{Concluding remarks}

In conclusion, the mechanism behind absence of homogenization in the random potential system is that the potential is unbounded from above and that the behavior of the system is dominated by the large fluctuations in the potential. In contrast, the Hamiltonian of unbounded random advection of gradient type is equivalent to a classical mechanics Hamiltonian with a potential which, though unbounded from below, is bounded from above, and so satisfies a homogenization principle. It is an interesting future work to unravel more delicate conditions of stochastic HJ homogenization for other Hamiltonians in unbounded random media. A related problem is to study Hamiltonians with unbounded temporal fluctuations. For reaction-diffusion fronts in incompressible random advection, temporal randomness is found to regularize the dominance of extreme events and promote mixing; hence the speed of propagation is asymptotically a deterministic constant $[13,7,9]$. It is conceivable that similar results (or homogenization) hold for HJ equations in unbounded time-dependent random media under suitable conditions.

Acknowledgements. W. E is supported in part by NSF grant DMS04-07866 and DOE grant DE-FG02-03ER25587. J.W. is grateful for the hospitality and the Oden research fellowship at the Institute of Computational Engineering and Sciences at the University of Texas at Austin. J.X is partially supported by NSF grant DMS0549215 .

\section{REFERENCES}

[1] R. Adler, The Geometry of Random Fields, John Wiley and Sons, 1980.

[2] R. Durret, Probability: Theory and Examples, Duxbury, 1994.

[3] L. C. Evans, Partial Differential Equations, Graduate Studies in Mathematics, American Mathematical Society, Providence, R.I, 1998.

[4] M. Freidlin, Functional Integration and Partial Differential Equations, Ann. Math. Stud. Princeton University Press, Princeton, NJ, 109, 1985.

[5] I. Karatzas and S. Shreve, Brownian Motion and Stochastic Calculus, Springer-Verlag, 1991.

[6] M.R. Leadbetter, G. Lindgren and H. Rootzn, Extremes and Related Properties of Random Sequences and Processes, Springer Series in Statistics, New York, Springer-Verlag, 1983.

[7] J. Nolen and J. Xin, Variational principle of KPP front speeds in temporally random shear flows with applications, Comm. Math. Phys., 269, 493-532, 2007.

[8] J. Nolen and J. Xin, KPP Fronts in a one dimensional random drift, preprint, 2007.

[9] J. Nolen and J. Xin, Asymptotic spreading of KPP reactive fronts in incompressible space-time random flows, preprint, 2007.

[10] F. Rezakhanlou and J. Tarver, Homogenization for stochastic Hamilton-Jacobi equations, Arch. Rational Mech. Anal., 151, 277-309, 2000.

[11] P.E. Souganidis, Stochastic homogenization of Hamilton-Jacobi equations and some applications, Asymptotic Analysis, 20, 1-11, 1999.

[12] J. Wehr and J. Xin, Front speed in the Burgers equation with a random flux, J. Stat. Physics, 88(3/4), 843-871, 1997.

[13] J. Xin, KPP front speeds in random shears and the parabolic Anderson problem, Meth. Appl. Anal., 10(2), 191-198, 2003.

[14] J. Xin, Front propagation in heterogeneous media, SIAM Review, 42(2), 161-230, 2000.

[15] V. Yakhot, Propagation velocity of premixed turbulent flames, Comb. Sci. Tech., 60, 191, 1988. 